\title{
The characteristics of a pneumatic muscle
}

\author{
Dawid Pietrala \\ Department of Automation and Robotics, Kielce University of Technology, 25-314 Kielce, Poland
}

\begin{abstract}
The article presents static and dynamic characteristics of pneumatic muscles. It presents the structure of the laboratory stand used to test pneumatic muscles. It discusses the methodology for determination of static and dynamic characteristics. The paper also illustrates characteristics showing the relationship of pneumatic muscles length and operating pressure, at a constant loading force (isotonic characteristics). It presents characteristics showing the relationship of pneumatic muscles shortening and values of loading forces, at a constant operational pressure (isobaric characteristics). It also shows the dependence of force generated by the muscle on the operating pressure, at a constant value of pneumatic muscles shortening (isometric characteristics). The paper also presents dynamic characteristics of a pneumatic muscle showing the response of an object to a gradual change in the operating pressure, at a constant loading force acting on the pneumatic muscle.
\end{abstract}

\section{Introduction}

The pneumatic artificial muscle is a type of single acting pneumatic actuator. It is made by placing a stretchable bladder inside of the elastic braid. Both elements are connected by permanent ends. One of them is used to supply pressurized air into the muscle. The increase in internal pressure of the muscle causes an increase in its diameter, and thus its shortening. The change in length of the muscle can be used as an operating movement in order to drive the machinery. The return of the muscle to the starting position must be achieved by the application of external forces (spring return, the force of gravity or the second pneumatic artificial muscle) $[1,2]$.

\section{The methodology for determining the characteristics of pneumatic muscles}

The work of a pneumatic muscle is characterized by three basic parameters: internal pressure $P$, loading force $F$ and relative shortening $\varepsilon$. Relative shortening, or the degree of muscle shortening, is expressed as the percentage dependence of the change in the muscle length with respect to its nominal length $[1,3,4]$.

$$
\varepsilon=\frac{L-L_{n}}{L_{n}} \cdot 100 \%
$$

where:

$\varepsilon$ - relative shortening of the muscle,

$L$ - actual length of the muscle,

$L_{n}$ - nominal length of the muscle.

The static isotonic characteristic shows the dependence of muscle shortening $\varepsilon$ on changes in the internal pressure $p$ at a constant loading force $F=$ const. This characteristic was determined in the following manner: one end of the muscle was securely fixed to the stationary base. The other end of the muscle was affected by a known constant loading force, acting in the muscle axis. The return of that force was directed in a way that stretched the muscle. Then the internal pressure of the muscle was increased and the value of shortening degree was read. The pressure was reduces after reaching the maximum pressure and the value of shortening degree was read again. This procedure was repeated for successive values of loading forces.

The static isobaric characteristic shows the dependence of muscle shortening $\varepsilon$ on changes in the loading force $F$, at a constant internal pressure $p=$ const . When determining this characteristic one end of the muscle was also permanently immobilized. The muscle was subjected to a known constant internal pressure. Then the loading force of the muscle was gradually increased and the shortening degree was read. The force was gradually reduced after reaching the maximum loading force and the value of shortening degree was read again. This procedure was repeated for successive values of internal pressure of the muscle $[1,5,6]$.

The static isometric characteristic shows the dependence of loading force $F$ on changes in the internal pressure $p$, at a constant shortening of the muscle $\varepsilon=$ const . This characteristic was determined in the following manner. One end of the muscle was permanently immobilized. The other end of the muscle was immobilized in a way that made it possible to read the actual length of the muscle, and thus to read the actual shortening degree of the muscle. Then the internal pressure of the muscle was gradually increased and the actual force generated by the muscle was read. The pressure was gradually reduced after reaching the

Corresponding author: dpietrala@tu.kielce.pl 
maximum value and the actual force generated by the muscle was read again. This procedure was repeated for successive shortening degrees of the muscle.

The dynamic characteristics of pneumatic muscles illustrate shortening of the muscle $\varepsilon$ in a time function $t$, as a response of the object to a gradual change of internal pressure of the muscle $p$, at a constant loading force $F=$ const [7-10] .

\section{The laboratory stand used to test pneumatic muscles}

The characteristics presented in the article were determined on a laboratory stand for testing pneumatic muscles. This stand was designed and made by the author of this article. It is adapted to conduct studies on an individual muscle or pairs of muscles operating antagonistically. The stand makes it possible to determine static and dynamic characteristics of pneumatic muscles. The scheme and actual view of the stand was shown in fig. 1. The stand includes the supporting structure, linear guides that adjust the stand to the length of the tested muscle and bearing block with gear belt drive, turning the plane motion of the muscle on the rotary motion. An important element of the stand is the AC MT-C06401C2NT3D servomotor from Servotronix with the CDHD-0032AAP1 servo controller and a precise planetary gear [11-14]. It is used to load the axis of a gear belt drive with a known torque, and thereby to load the muscle with the force of known value. The chosen servomotor has the capability of producing a continuous torque with the value of $26 \mathrm{Nm}$, and an instantaneous torque with the value of up to 78 $\mathrm{Nm}$. This translates into a continuous loading force of the muscle with the value of up to $812 \mathrm{~N}$, or an instantaneous torque with the value of up to $2436 \mathrm{~N}$. In addition, the stand was equipped with a strain gauge with a measuring range of $\pm 1000 \mathrm{~N}$ with an analogue voltage output. The change in internal pressure is achieved by means of a piezoelectric proportional pressure valve Hoerbiger Tecno Plus with an analogue voltage input. It allows to change the pressure in range of $0-10$ bars. The measurement of the muscle length is implemented as a measurement of the angle of rotation of the gear belt drive by means of an optical absolute encoder Posital/Fraba OCD-S101G-1416-S060-CAW with a resolution of 16 bit with the SSI digital output. The control of stand components and data acquisition is performed by means of an electronic circuit with the STM32F407VGT6 microcontroller equipped with circuits of 16 analogue inputs and 16 analogue outputs with a resolution of 16 bit each, and an interface supporting up to 6 sensors with the SSI output [15-18].
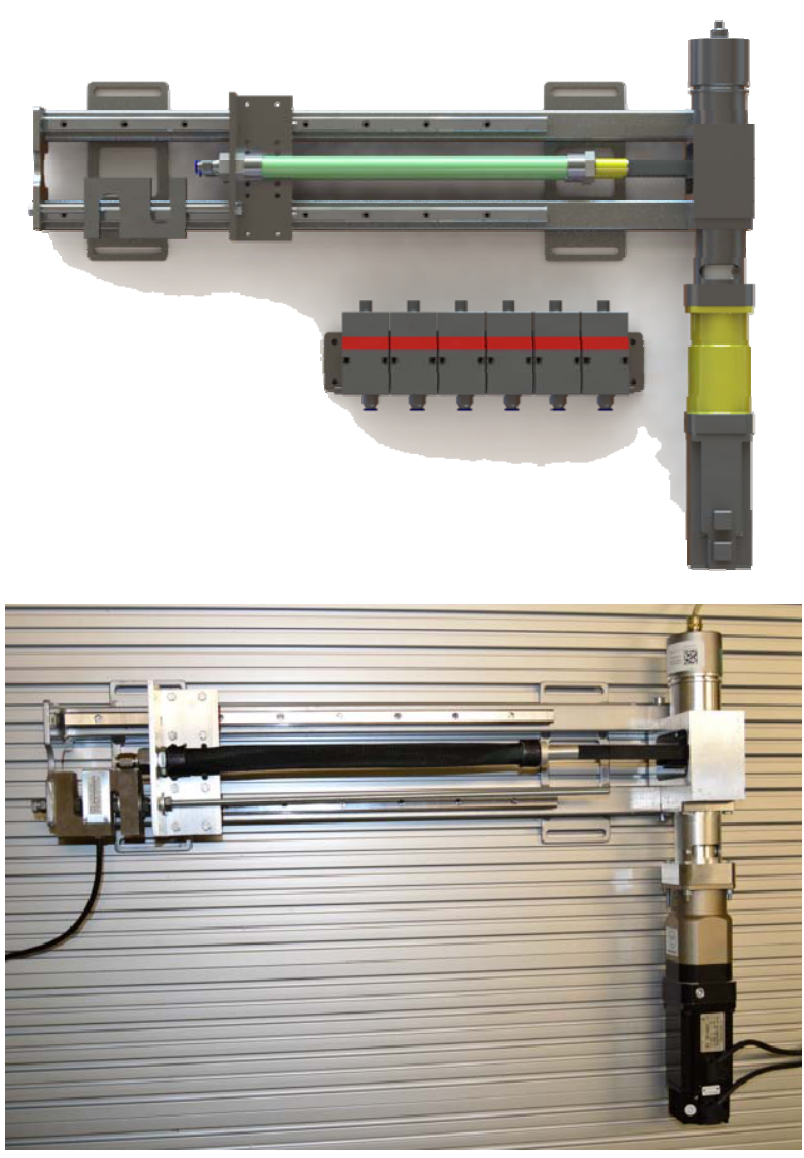

Fig. 1 The stand used to test pneumatic muscles

\section{Static and dynamic characteristics of pneumatic muscles}

The paper presents static characteristics: isotonic, isobaric and isometric as well as dynamic characteristics of pneumatic muscles. These dependencies were designated on the laboratory stand described above, and the tested object was a pneumatic artificial muscle made according to the developer's project. The muscle contains a latex bladder placed on an elastic braid and modelled on pneumatic muscles from Shadow Air Muscle, which means that the braid is connected to the bladder only at the ends of the muscle. The nominal length of the muscle is $350 \mathrm{~mm}$.

Fig. 2 and fig. 3 present a number of static and isotonic characteristics of the tested muscle. These dependencies were designated for loading forces $F=$ const in the range from $50 \mathrm{~N}$ to $550 \mathrm{~N}$. Each characteristic was designated by gradual increase of the internal pressure $P$ in the muscle from 0bar to 3 bars in 0.1 bar increments $[3,4,19]$. 


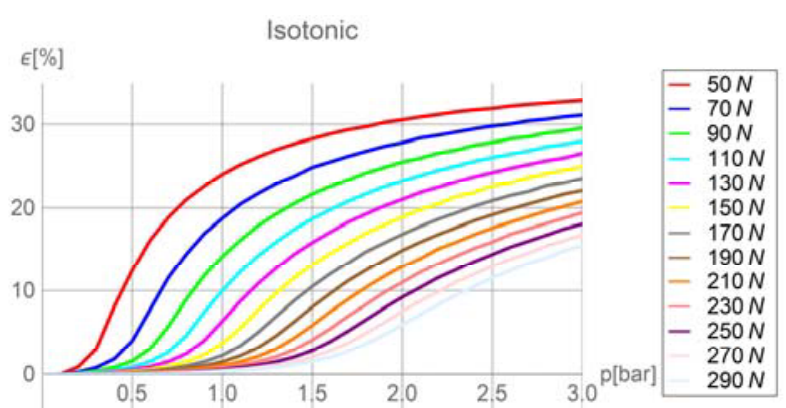

Fig. 2 Isotonic characteristics for constant loading forces from $50 \mathrm{~N}$ to $290 \mathrm{~N}$ at a pressure increase.

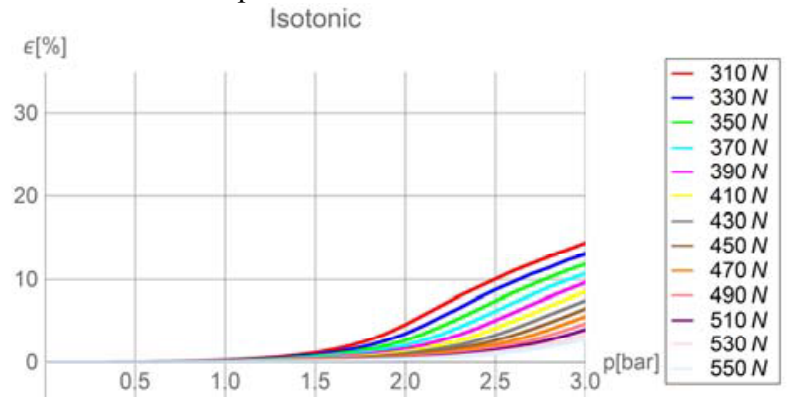

Fig. 3 Isotonic characteristics for constant loading forces from $310 \mathrm{~N}$ to $550 \mathrm{~N}$ at a pressure increase.

Fig. 4 and fig. 5 present a number of static isotonic characteristics of the tested muscle for the same loading forces $F=$ const . Each characteristic was designated by gradual decrease of the internal pressure $p$ in the muscle from 3 bars to 0 bar in 0.1 bar increments.

\section{Isotonic}

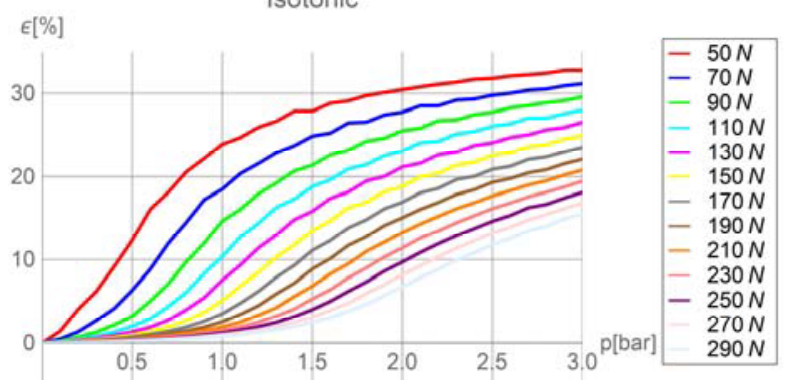

Fig. 4 Isotonic characteristics for constant loading forces from $50 \mathrm{~N}$ to $290 \mathrm{~N}$ at a pressure decrease.

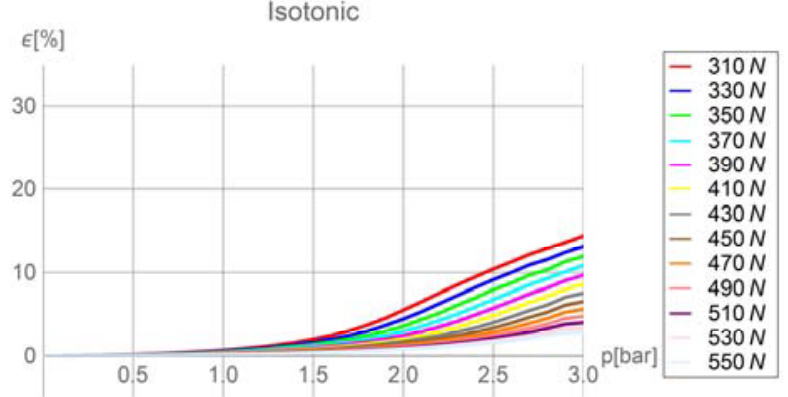

Fig. 5 Isotonic characteristics for constant loading forces from $310 \mathrm{~N}$ to $550 \mathrm{~N}$ at a pressure decrease.

When comparing two figures one can see the difference between the corresponding characteristics which is the hysteresis of a working muscle. This means that when the muscle shortens, it moves in a different way than when it increases its length. The hysteresis for the two isotonic characteristics can be seen in fig. 6 and fig. 7 .

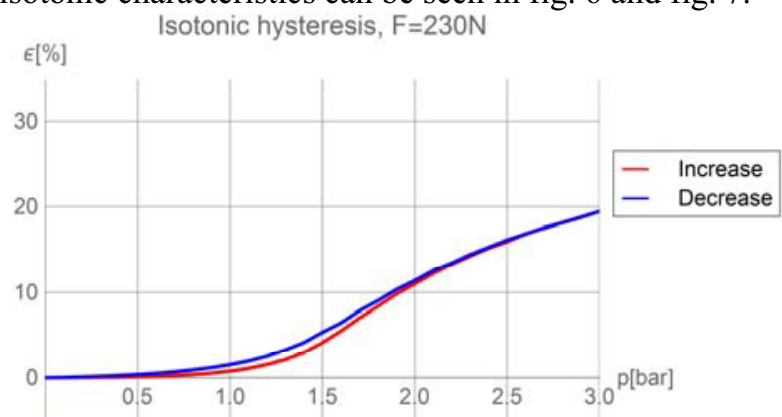

Fig. 6 Hysteresis for the selected isotonic characteristic.

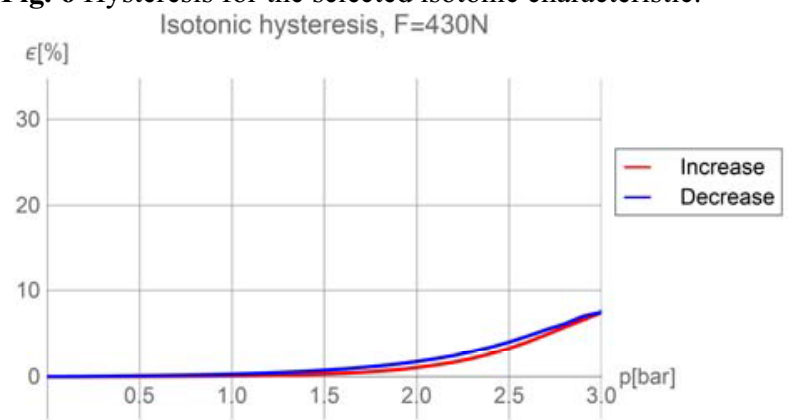

Fig. 7 Hysteresis for the selected isotonic characteristic.

Fig. 8 and fig. 9 present a series of static isobaric characteristics. These dependencies were designated for constant internal pressures of the muscle $p=$ const in the range from 0bar to 3bars. Each characteristic was designated by gradual increase of the loading force $F$ of the muscle from $50 \mathrm{~N}$ to $550 \mathrm{~N}$ in $20 \mathrm{~N}$ increments.

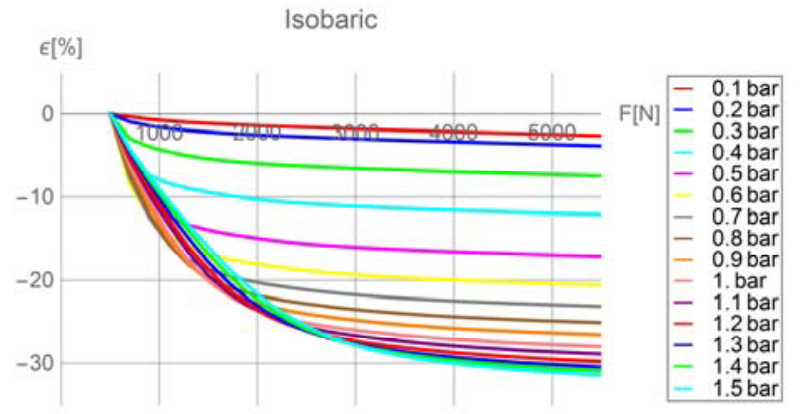

Fig. 8 Isobaric characteristics for constant internal pressures from 0.1 bar to 1.5 bar at a force increase.

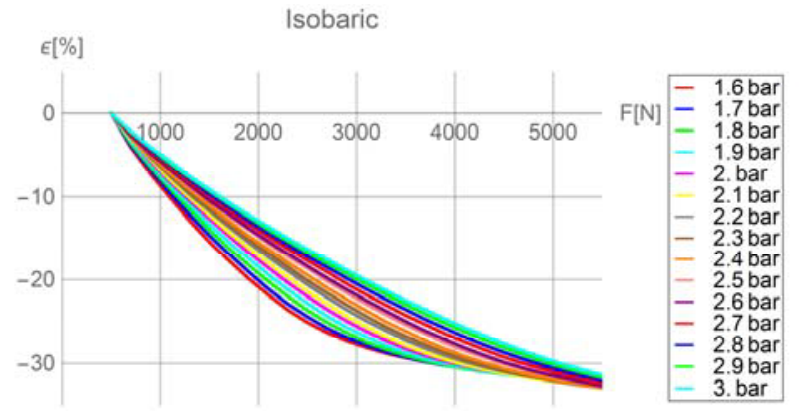

Fig. 9 Isobaric characteristics for constant internal pressures from 1.6 bar to 3 bars at a force increase.

Fig. 10 and fig. 11 present a number of static isobaric characteristics of for the same constant internal pressures $p=$ const . Each characteristic was designated 
by gradual decrease of loading forces $F$ in the range from $550 \mathrm{~N}$ to $50 \mathrm{~N}$ in $20 \mathrm{~N}$ increments.

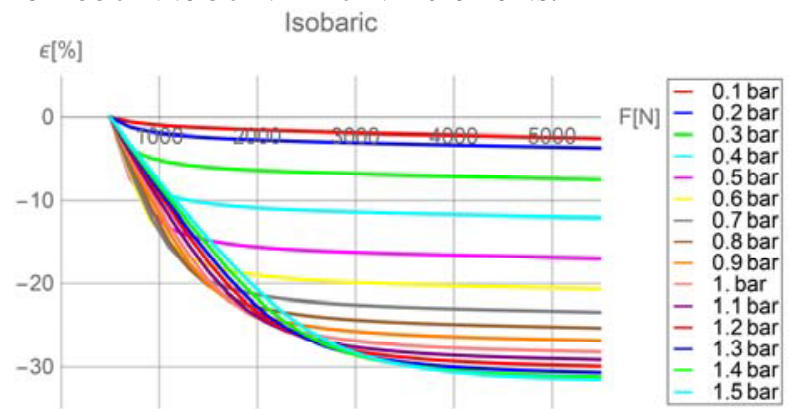

Fig. 10 Isobaric characteristics for constant internal pressures from 0.1 bar to 1.5 bar at a force decrease.

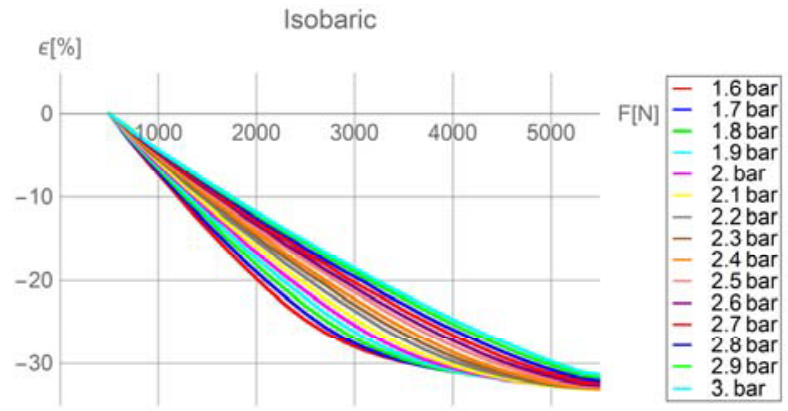

Fig. 11 Isobaric characteristics for constant internal pressures from 1.6 bar to 3 bars at a force decrease.

As in the case of isotonic characteristics, the isobaric characteristics also show hysteresis between the corresponding dependencies. Hysteresis for two exemplary isobaric characteristics was shown in fig. 12 and fig. 13.

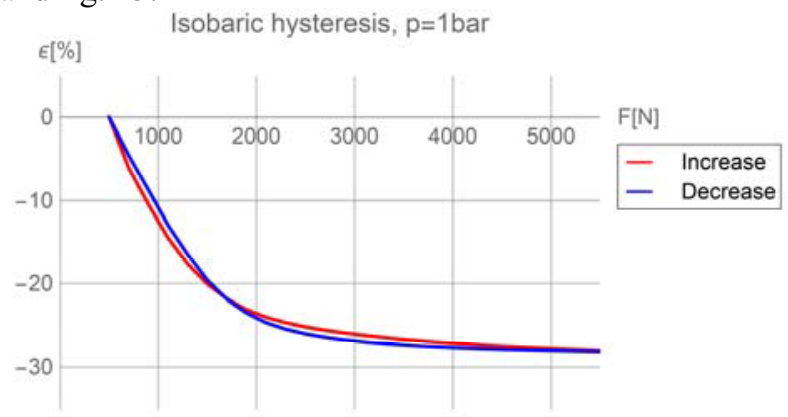

Fig. 12 Hysteresis for the selected isobaric characteristic.

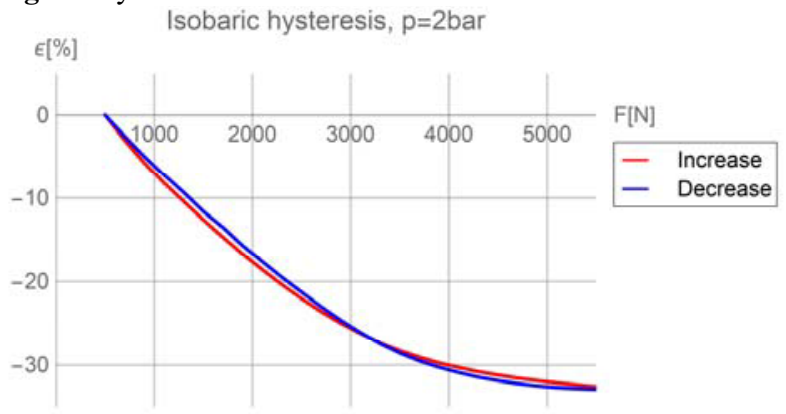

Fig. 13 Hysteresis for the selected isobaric characteristic.

The paper also presents isometric characteristics showing the dependence of force $F$ produced by the muscle in a function of internal pressure $p$ at a constant shortening degree $\varepsilon=$ const . Fig. 14 and fig. 15 present a series of isometric characteristics. These dependencies were designated for constant values of the muscle shortening. The muscle length varied from a nominal value of $350 \mathrm{~mm}$ to $250 \mathrm{~mm}$ in $5 \mathrm{~mm}$ increments, which provided the degree of muscle shortening in the range from $0 \%$ to $35.71 \%$. Each characteristic was designated by gradual increase of the internal pressure $p$ in the muscle from 0 bar to 3 bars, in 0.1 bar increments.

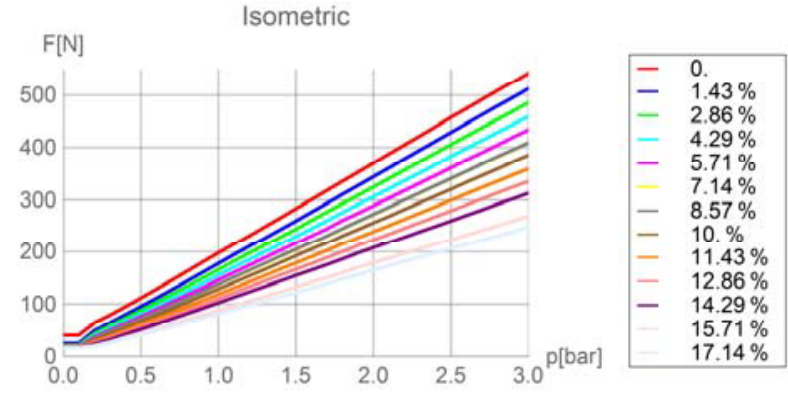

Fig. 14 Isometric characteristics for constant shortening of the muscle from $0 \%$ to $17.14 \%$ at a pressure increase.

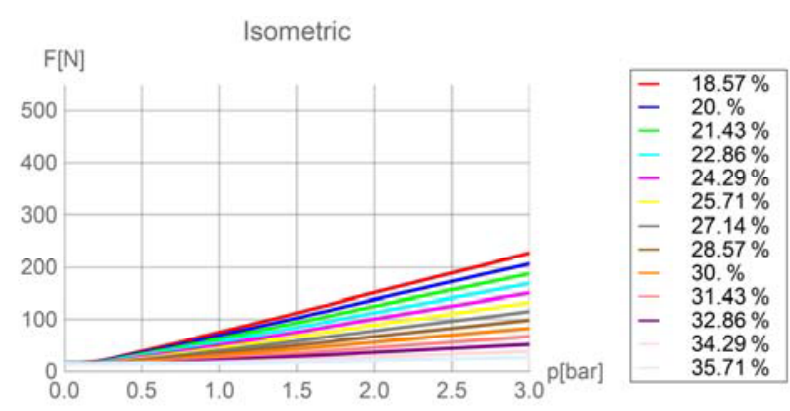

Fig. 15 Isometric characteristics for constant shortening of the muscle from $18.57 \%$ to $35.71 \%$ at a pressure increase.

Fig. 16 and fig. 17 present a number of static isometric characteristics of the tested muscle for the same values of the shortening degree $e=$ const . Each characteristic was designated by gradual decrease of the internal pressure $p$ in the muscle from 3 bars to 0 bar in 0.1 bar increments.

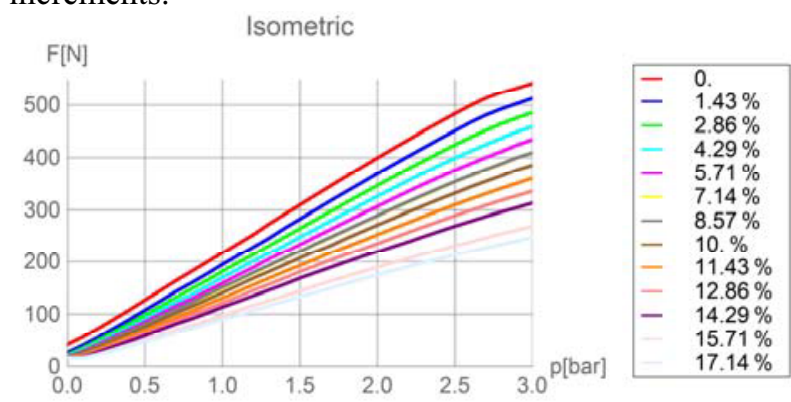

Fig. 16 Isometric characteristics for constant shortening of the muscle from $0 \%$ to $17.14 \%$ at a pressure decrease. 


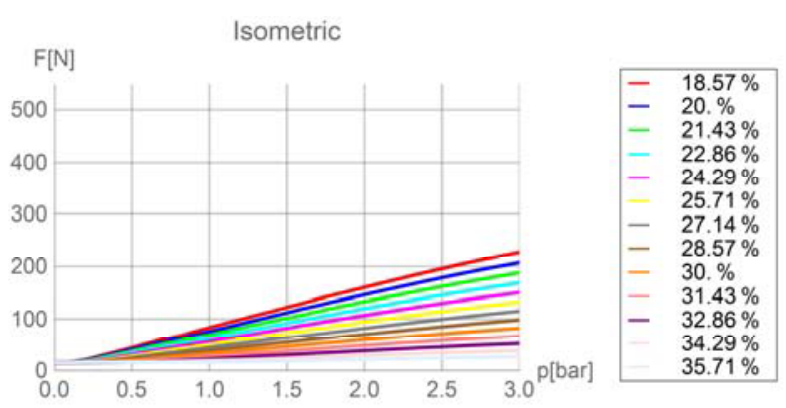

Fig. 17 Isometric characteristics for constant shortening of the muscle from $18.57 \%$ to $35.71 \%$ at a pressure decrease.

In the case of isometric characteristics one can also observe the difference between the corresponding characteristics which is the hysteresis of a working muscle. Hysteresis for two selected isometric characteristics can be seen in fig. 18 and fig. 19.

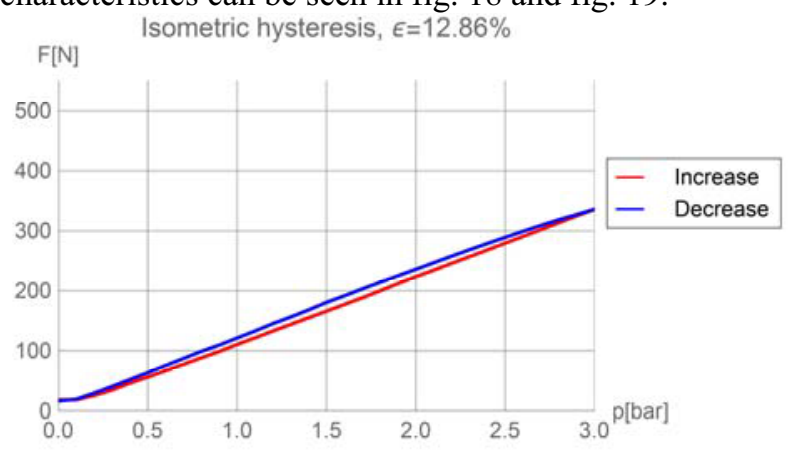

Fig. 18 Hysteresis for the selected isometric characteristic.

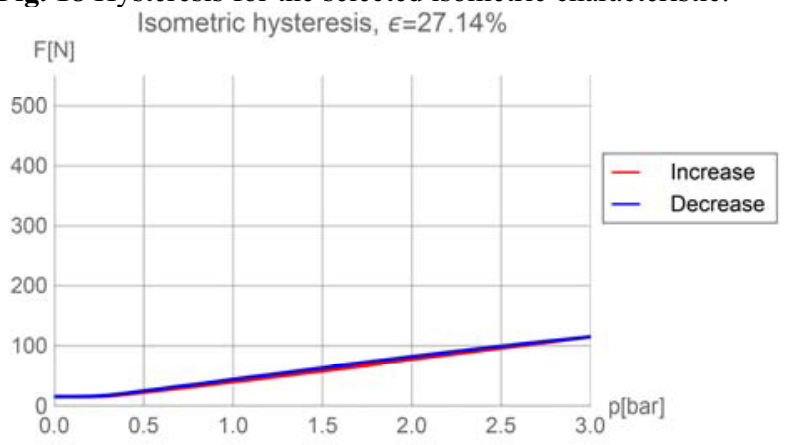

Fig. 19 Hysteresis for the selected isometric characteristic.

The paper also presents dynamic characteristics of pneumatic muscles. These dependencies show the muscle shortening $\varepsilon$ as the response of the object to a gradual change in the internal pressure $p$ from 0 bar to the set pressure value, at constant values of loading forces $F=$ const. Sampling period when determining dynamic characteristics was $5 \mathrm{~ms}$. fig. 20 -fig. 23 present the selected dynamic characteristics of pneumatic muscles $[3,20]$.

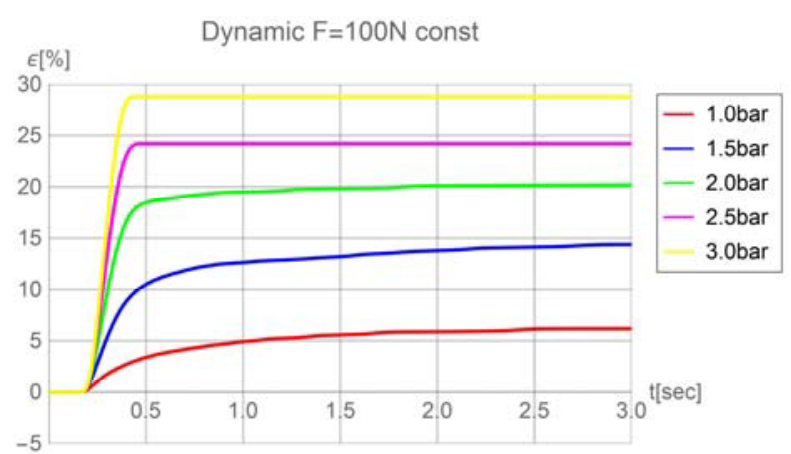

Fig. 20 The muscle response to gradual changes in pressure from 0 bar to the indicated value at a constant loading force $\mathrm{F}=100 \mathrm{~N}$

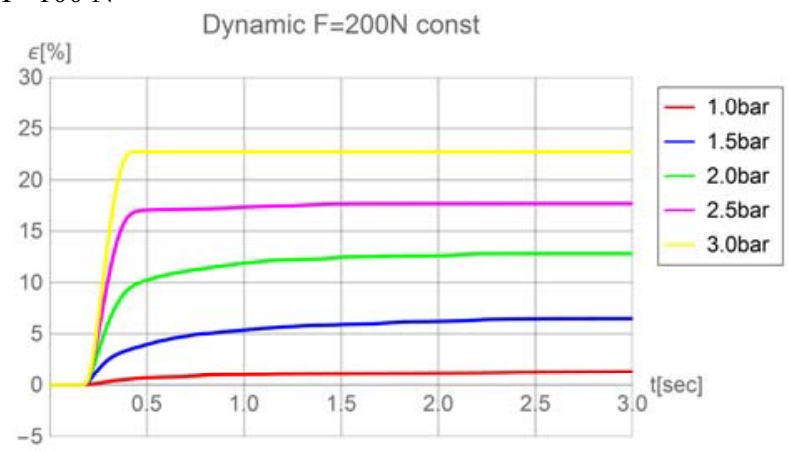

Fig. 21 The muscle response to gradual changes in pressure from 0 bar to the indicated value at a constant loading force $\mathrm{F}=200 \mathrm{~N}$

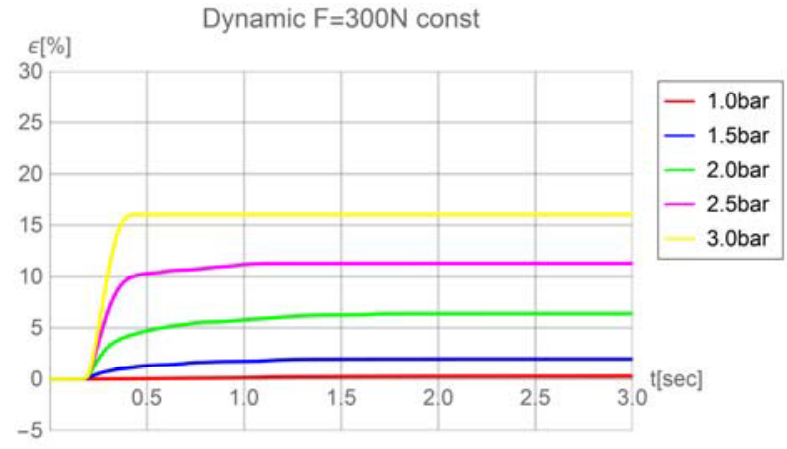

Fig. 22 The muscle response to gradual changes in pressure from 0 bar to the indicated value at a constant loading force $\mathrm{F}=300 \mathrm{~N}$

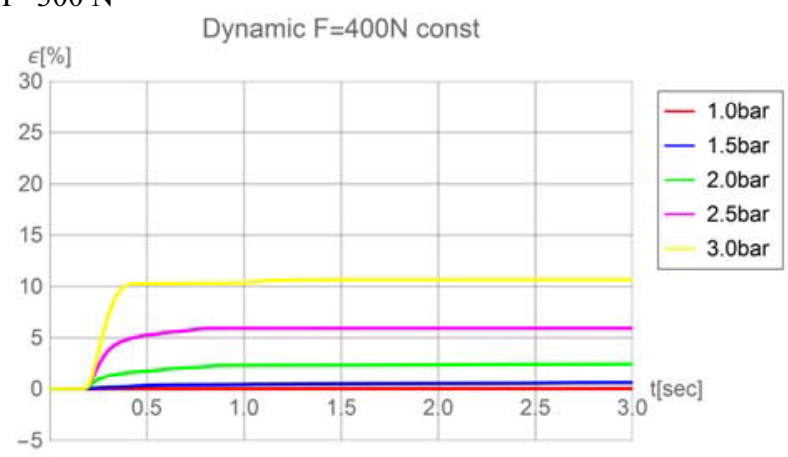

Fig. 23 The muscle response to gradual changes in pressure from 0 bar to the indicated value at a constant loading force $\mathrm{F}=400 \mathrm{~N}$

\section{Conclusions}

Pneumatic muscles seen as drive elements have a number of features that distinguish them from 
conventional drives. The total tightness, lack of stick-slip phenomenon and generation of smooth movements have a positive impact on the ability to use the muscles as drive elements. Non-linear nature of the muscles hinders the precise control of such drives. For this purpose, it is extremely important to know all the dependencies that bind basic parameters of pneumatic muscles: the loading force, internal pressure and shortening degree.

The article presents basic concepts concerning pneumatic muscles. It presents methodologies for determining static and dynamic characteristics of muscles. It describes the structure of laboratory stand used to test pneumatic muscles. The paper also presents static and dynamic characteristics of an exemplary pneumatic muscle.

\section{References}

1. P.A. Laski, J.E. Takosoglu, S. Blasiak, (ACAD SCI CZECH REPUBLIC, INST THERMOMECHANICS, DOLEJSKOVA 5, PRAGUE 8, 182 00, CZECH REPUBLIC, 2014).

2. D.S. Pietrala, (ACAD SCI CZECH REPUBLIC, INST THERMOMECHANICS, DOLEJSKOVA 5, PRAGUE 8, 182 00, CZECH REPUBLIC, 2016).

3. J.E. Takosoglu, P.A. Laski, S. Blasiak, G. Bracha, D. Pietrala, MEASUREMENT \& CONTROL. 49 (2) (2016), 62-71.

4. P.A. Laski, J.E. Takosoglu, S. Blasiak, Robotics and Autonomous Systems. (2014),.

5. P.A. Laski, (ACAD SCI CZECH REPUBLIC, INST THERMOMECHANICS, DOLEJSKOVA 5, PRAGUE 8, 182 00, CZECH REPUBLIC, 2016).

6. J.E. Takosoglu, P.A. Laski, S. Blasiak, (ACAD SCI CZECH REPUBLIC, INST THERMOMECHANICS, DOLEJSKOVA 5, PRAGUE 8, 182 00, CZECH REPUBLIC, 2014).

7. S. Blasiak, Journal of Thermal Science and Technology. 10 (1) (2015), JTST0016-JTST0016.

8. S. Blasiak, C. Kundera, Procedia Engineering. 39 (2012), 315-326.

9. S. Blasiak, C. Kundera, J. Bochnia, Procedia Engineering. 39 (2012), 366-378.

10. S. Blasiak, A. Pawinska, International Journal of Heat and Mass Transfer. 90 (2015), 710-718.

11. D. Janecki, J. Zwierzchowski, MEASUREMENT SCIENCE \{\&\} TECHNOLOGY. 26 (8) (2015),

12. J. Zwierzchowski, (ACAD SCI CZECH REPUBLIC, INST THERMOMECHANICS, DOLEJSKOVA 5, PRAGUE 8, 182 00, CZECH REPUBLIC, 2016).

13. D. Janecki, J. Zwierzchowski, L. Cedro, BULLETIN OF THE POLISH ACADEMY OF SCIENCESTECHNICAL SCIENCES. 63 (3) (2015), 771-779.

14. E. Miko, L. Nowakowski, (ELSEVIER SCIENCE BV, SARA BURGERHARTSTRAAT 25, PO BOX 211, 1000 AE AMSTERDAM, NETHERLANDS, 2012).

15. D. Janecki, J. Zwierzchowski, (IMEKO, PO BOX 457, H-1371 5 BUDAPEST, HUNGARY, 2009).
16. D. Janecki, L. Cedro, J. Zwierzchowski, METROLOGY AND MEASUREMENT SYSTEMS. 22 (2) (2015), 289-302.

17. B. Nowakowski, A.L. Kawczynski, A. Kolbus, A. Lemarchand, EUROPEAN PHYSICAL JOURNAL B. 84 (1) (2011), 137-145.

18. A. Nowakowski, M. Cybulski, A. Sliwczynski, A. Chil, Z. Teter, P. Seroczynski, et al., BMC CANCER. 15 (2015),

19. J.E. Takosoglu, R.F. Dindorf, P.A. Laski, INTERNATIONAL JOURNAL OF ADVANCED MANUFACTURING TECHNOLOGY. 40 (3-4) (2009), 349-361.

20. G.F. Bracha, (ACAD SCI CZECH REPUBLIC, INST THERMOMECHANICS, DOLEJSKOVA 5, PRAGUE 8, 182 00, CZECH REPUBLIC, 2016). 